\title{
AN INNOVATIVE DAY HOSPITAL DEDICATED TO NURSING HOME RESIDENT: A DESCRIPTIVE STUDY OF 1306 RESIDENTS REFERRED BY THEIR PHYSICIANS
}

\author{
C. LAFFON DE MAZIÈRES ${ }^{1,2}$, M. ROMAIN ${ }^{1}$, S. HERMABESSIÈRE $^{1}$, G. ABELLAN ${ }^{1,2}$, S. GERARD $^{1}$,

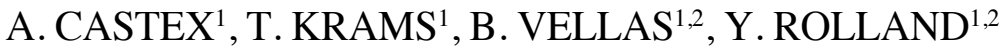

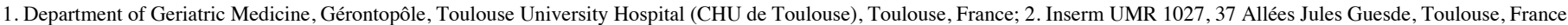

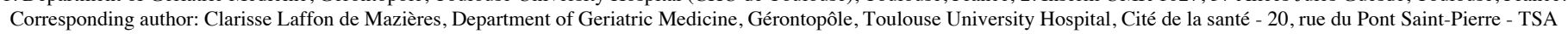
60033 - 31059 Toulouse cedex 9, France. E-mail address : laffondemazieres.c@chu-toulouse.fr. Tel: +33 (0)5 61777046

\begin{abstract}
Background: The transfer rate of residents from nursing homes (NH) to emergency rooms is high. These transfers are often inappropriate but also potentially avoidable. Recent studies have shown that in terms of methods for training NH teams, proposals for improvement of the healthcare sector must be organized. Given this observation, Gérontopôle de Toulouse (France) opened in October 2015, a responsive day hospital dedicated to $\mathrm{NH}$ residents (DH NH). This day hospital is characterized by its vocation, exclusively dedicated to NH residents and its ability to provide patient care within a short period of time. Objectives: The purpose of this day hospital is twofold: (1) decrease the rate of inappropriate transfers for $\mathrm{NH}$ residents by offering general practitioners and $\mathrm{NH}$ teams quick access to expert advice, blood tests and radiological examinations during hospitalizations and care adapted to the characteristics of $\mathrm{NH}$ residents; (2) potentially reduce avoidable transfers to emergency rooms and hospitalizations by taking action to prevent acute decompensation in residents, but also for the education and training of $\mathrm{NH}$ healthcare teams. This manuscript aims to describe the arrangements put in place and the characteristics of the residents collected after two years of activity. Design: Retrospective descriptive study. Setting: Gérontopôle of Toulouse, France. Participants: 1306 residents have been consulted at the DH NH. Measurements: Referring physicians (treating physicians, coordinating physician or emergency room physicians) send a standardized hospitalization request form to the day hospital by fax or email indicating the reason for the request, specialist opinion(s) desired and additional required examination(s). A gerontological assessment was conducted and anamnesis data was collected for each resident, on the very day of their coming to the DH NH. Results: In 2 years, 1306 residents from $120 \mathrm{NHs}$ were sent to the DH NH. The mean age was 86.23 \pm 7.05 years and the majority of patients were women $(n=941,72.22 \%)$, dependent (median ADL at 2.75, [1.25-4.5]) and malnourished $(821,63.25 \%)$. In the 3 months prior to their visit to the day hospital, $668(57.14 \%)$ residents had been hospitalized, and one-quarter $(n=336,25.72 \%)$ had been transferred to emergency rooms. The main reasons for hospitalization included assessment of cognitive disorders $(\mathrm{n}=336,17.52 \%)$, assistance in managing behavioral disorders $(n=297,15.48 \%)$ and bedsores and slow wound healing $(n=223,11.63 \%)$. Conclusion: Our experience over a 2-year period suggests that the $\mathrm{DH} \mathrm{NH}$ could be a practical response to the problem of inappropriate and avoidable transfers of $\mathrm{NH}$ residents to emergency rooms. This innovation could easily be utilized in other hospitals.
\end{abstract}

Key words: Elderly, nursing home, emergency transfers, avoidable hospitalizations.

\section{Introduction}

The transfer rate of residents from nursing homes $(\mathrm{NH})$ to emergency rooms is high. In the United States, $25 \%$ of residents are transferred to emergency rooms at least once per year and $10 \%$ of residents 2 or more times (1). In France, approximately $50 \%$ of residents are hospitalized each year and more than half of the hospitalizations take place after a transfer to emergency rooms (2). In the United States, each resident is hospitalized nearly 2 times per year (3). These data reflect the great vulnerability of $\mathrm{NH}$ residents, but they also generate concerns because the transfer of $\mathrm{NH}$ residents to emergency rooms exposes them to multiple risks, such as confusion, falls, bedsores, functional decline, and mortality (4-6). In addition, these transfers cause organizational dysfunction within

emergency units, which are often overcrowded and ill-equipped to care for elderly, dependent and often mentally ill subjects. Trips to the emergency room are often longer for $\mathrm{NH}$ residents than those of young subjects (7-10).

Limiting the use of emergency rooms to patients who need it would be an unacceptable missed opportunity. However, many studies show that in 20 to $67 \%$ of cases the transfer of residents are potentially avoidable (11-15). Some data reports that more than two-thirds of residents have no severe clinical sign upon arrival to emergency rooms resulting in more than one-half of transfers to emergency rooms not requiring subsequent hospitalization (3). In addition, nearly $20 \%$ of residents leave without any diagnosis. These data suggest that alternatives must be organized to limit the number of potentially avoidable transfers to emergency rooms.

Published online September 19, 2018, http://dx.doi.org/10.1007/s12603-018-1106-5 


\section{THE JOURNAL OF NUTRITION, HEALTH \& AGING@}

INTERACT (Interventions to Reduce Acute Care Transfers), a program for the improvement of clinical practices, was arranged in the United States in order to limit the transfer of $\mathrm{NH}$ residents to hospitals. This program, which is based on improving the identification, assessment and management of acute medical situations by the NH healthcare team, has not yet demonstrated its efficacy on decreasing hospitalizations (16). The results of INTERACT suggest that in terms of training NH teams, proposals for improving the healthcare sector must be organized for residents, because despite the actions carried out within the $\mathrm{NH}$, the healthcare teams often remain confronted with acute medical situations requiring additional tests and expert advice. The risks associated with transfers to emergency rooms are well known to treating physicians and $\mathrm{NH}$ healthcare teams, but the lack of quick response from traditional hospital services or other alternatives often results in an inappropriate use of emergency rooms $(11,17)$. To date, no other sector offers the simplicity and rapid access to technical platforms, expert advice and monitoring of emergency services. However, in the IQUARE study, we have shown that collaboration between the $\mathrm{NH}$ healthcare team and geriatricians decreases the number of emergency room transfers for residents (18). Other studies also show that apart from the characteristics of residents (multiple comorbidities (19), degree of dependence), the organization within the $\mathrm{NH}$ (care protocol, partnership with the hospital) determines the transfer rate of residents to emergency rooms $(17,20)$.

Given this observation, Gérontopôle de Toulouse (France) opened in October 2015, a responsive day hospital dedicated to $\mathrm{NH}$ residents $(\mathrm{DH} \mathrm{NH})$. This day hospital is characterized by its vocation, exclusively dedicated to $\mathrm{NH}$ residents and its ability to provide patient care within a short period of time. The purpose of this day hospital is twofold: (1) decrease inappropriate transfers to emergency rooms by offering general practitioners and $\mathrm{NH}$ teams quick access to expert advice, blood tests and radiological examinations during hospitalizations and care adapted to the characteristics of NH residents; (2) reduce potentially avoidable transfers to emergency rooms and hospitalizations by taking action to prevent acute decompensation in residents, but also for the education and training of $\mathrm{NH}$ healthcare teams.

In this article, we present the organization of the responsive day hospital dedicated to $\mathrm{NH}$ residents and the main characteristics and reasons for sending residents collected since its opening.

\section{Method}

The innovative day hospital dedicated to $\mathrm{NH}$ residents occupies a central position within the care units of the Geriatrics Department of Toulouse, located in the Centre Hospitalier Universitaire (CHU) de Toulouse (France). The admission capacity is approximately 4 residents per day. The capacity of the home has increased in one year to 6 residents per day, 5 days per week. A full-time geriatrician is on staff at the day hospital as well as other medical specialists, who are on-call and respond to requests as needed. Nurses work at a rate of 1.8 full-time equivalents (FTE) (i.e. 35 hours/week) and caregivers at 1 FTE. Nurses provide preventive, curative or palliative care to residents and are also involved in scheduling examinations, which will benefit the residents during their hospitalization as well as the convening of residents. The premises consist of one room per patient (bed and bathroom), a treatment room, scheduling office and medical office.

The methods of sending to the day hospital are according to protocol. Residents can be directed to the $\mathrm{DH}$, either by the physician (treating physician or coordinating physician), or by emergency room physicians after transferring to the emergency room (post-emergency room care).

In practice, the requesters (treating physicians, coordinating physician or emergency room physicians) send a standardized hospitalization request form to the day hospital by fax or email indicating the reason for the request, specialist opinion(s) desired and additional required examination(s) (biological and/or radiological). Mention of the urgent nature of care is explicitly requested on this form in order to prioritize the requests. Request forms may be accompanied by a telephone call to the day hospital physician, particularly for urgent requests (hospitalization request within 48-72 hours) in order to the fulfill the request and accelerate the treatment. The request form is reviewed by the geriatrician at the day hospital. After approval of the request by the DH physician and obtaining of any additional information, the nurse organizes the resident's hospital day by contacting the various specialists required and making appointments for additional examinations. Finally, the nurse contacts the $\mathrm{NH}$ to communicate the date for the resident to come to the day hospital.

This procedure allows emergency room physicians to see $\mathrm{NH}$ residents who do not require traditional hospitalization after having been examined in the emergency room, but who could benefit from a geriatric opinion and/or another specialist as well as any additional examinations. This procedure enables early reorientation of residents to their $\mathrm{NH}$ after a short stay in the emergency room without having to wait for additional examinations, which are not performed in the emergency room.

Various opinions of medical specialists may be given at the day hospital (neurologist, cardiologist, urologist, pulmonologist, psychiatrist, rheumatologist, physical therapist, botulinum toxin specialist, infectologist, geriatric oncologist, palliative care/ pain specialist, specialist in bedsores and slow wound healing, dental surgeon). This singular service can be carried out in the resident's room without having to be transferred. In other words, the specialist visits the resident's bedside in the DH (for example, the heart ultrasound is performed in the resident's room in the $\mathrm{DH} \mathrm{NH}$ ) through the assistance of other physicians in the Geriatrics Department, but also in partnership with the physicians of other hospital departments who are aware of the constraints caused by moving dependent and often mentally 


\section{AN INNOVATIVE DAY HOSPITAL DEDICATED TO NURSING HOME RESIDENT}

ill residents. Medical imaging (CT and other scans) is carried out in a nearby building. Preferred time slots for X-rays have been defined with the Radiology Department in order to limit appointment delays.

Moreover, residents can benefit from other hospital services like any other hospitalized patient.

An organization is in place to ensure that the expertise of paramedics is available. When residents come to the $\mathrm{DH}$, they can benefit from the advice of a dietitian, an occupational therapist, a physiotherapist, and a speech therapist. Finally, the prescriptions of all residents are systematically reviewed by a pharmacist with an expertise in geriatrics. Proposals for prescription changes are systematically included in the discharge letter.

In order to modify the practices, an information campaign on this new activity was organized among the regional actors of geriatric care. Between March and October 2015, coordinating physician and/or coordinating nurse in NHs of the Toulouse healthcare field were notified by telephone of the opening of this day hospital and in traditional hospital discharge letters for residents when they returned to their $\mathrm{NH}$ or when they entered into a NH. Regionally, this new activity was the subject of an oral presentation at a regional congress (scientific meetings on aging with approximately 300 physicians and nurses). Nationally, we presented the 1-year assessment of this innovation at the Congrès National des Unités de Soins, d'évaluation et de prise en charge des patients Alzheimer (USPALZ) and the 36th Journées Annuelles de la Société Française de Gériatrie et Gérontologie.

The characteristics of all residents sent to the $\mathrm{DH} \mathrm{NH}$ during the first two years of activity were systematically entered in a standardized and prospective manner: data was collected for each resident, on the very day of their coming to the $\mathrm{DH}$ NH. The data collected were: age, sex, Charlson comorbidity index (21), degree of dependency assessed by the activities of daily living (ADL) scale on the hospitalization day and 3 months before (a score of 6 indicating total autonomy and 0 total dependence) (22). With the collection of weight (kilogram), height (meter), and serum albumin levels (gram/ liter), the nutritional status (malnutrition, yes/no) of each resident was defined according to the criteria of the Haute Autorité de Santé (23). The ability to walk was collected as a categorical variable (alone, with human assistance, with technical assistance, no ability to walk), as well as the presence of a sensory deficit (visual, auditory, auditory and visual, no deficit), falls during the month (yes/no), presence of pain (yes/ no) and bedsores (yes/no). The number of prescription drugs was analyzed as a continuous variable and the prescription of at least one neuroleptic drug (yes/no), benzodiazepine (yes/ no), or antidepressant (yes/no) was noted. The MMSE score was collected. The presence of behavioral disorders (yes/no) and, if applicable, the Neuropsychiatric Inventory (NPI) (24) score were collected. The Mini-Geriatric Depression Scale was analyzed as a continuous variable (a score of 0 indicates the high probability of no depression, and $\geq 1$ indicates a high probability of depression). Finally, we note whether the resident was sent directly by his/her treating physician at the day hospital (yes/no).

\section{Statistical analyses}

Data collection and statistical analyses were made by the referring physician of the $\mathrm{DH}$. The continuous variables were expressed by the mean and standard deviation (SD) (mean \pm SD) when they had a Gaussian distribution, otherwise by the median and deviation type (median [25-75]). Categorical variables were expressed in number and percentage. The analyses were carried out using Stata v14.2 software (StataCorp, College Station, TX, USA).

\section{Results}

\section{Characteristics of the residents}

The main characteristics of the $1306 \mathrm{NH}$ residents who were sent to the innovative day hospital for $\mathrm{NH}$ residents over a 2-year period are presented in Table 1 . The mean age was $86.23 \pm 7.05$ years and they were mainly women $(n=941$, $72.22 \%$ ). The residents were very dependent with a median ADL at 2.75, [1.25-4.5], and $821(63.25 \%)$ were malnourished. In the 3 months prior to their visit to the day hospital, 668 $(57.14 \%)$ residents had been hospitalized, and one-quarter $(n=336,25.72 \%)$ had been transferred to emergency rooms. 814 residents $(66.67 \%)$ were sent directly by the treating physician to the $\mathrm{DH} \mathrm{NH}$.

\section{Reasons for hospitalization}

Table 2 presents the reasons for hospitalization. Thus, 1918 different reasons for hospitalization were collected or 1.5 reasons per resident. The main reasons for hospitalization included assessment of cognitive disorders $(n=336,17.52 \%)$, assistance in managing behavioral disorders $(n=297,15.48 \%)$ and bedsores and slow wound healing $(\mathrm{n}=223,11.63 \%)$. Nutritional $(\mathrm{n}=197,10.27 \%)$, neurological $(\mathrm{n}=193,10.06 \%)$ and cardiac $(152,7.92 \%)$ reasons represented nearly one-third of the requests.

\section{Reactivity}

The time period of care was not collected for all 1306 residents. However, for 20 residents, chosen at random, for whom the treating physicians had mentioned the urgent nature of the request, the time period of care was 2.7 days.

The occupancy rate increased $77 \%$ in the opening year to 92.3\% in 2017 and was $93.2 \%$ from January to March 2018 . The number of NHs in the region that sent residents to the DH $\mathrm{NH}$ was 120 and among them, 80 sent residents to the DH NH several times. 


\section{Table 1}

Description of the characteristics of residents who have been consulted at the innovative day hospital dedicated to nursing home residents

\begin{tabular}{|c|c|}
\hline Characteristics $(n=1306)$ & $\begin{array}{c}\text { Mean } \pm \text { SD or median } \\
{[25-75] \text { or } n(\%)}\end{array}$ \\
\hline Age $(y), n=1301$ & $86.23 \pm 7.05$ \\
\hline Gender (female), $\mathrm{n}=1303$ & $941(72.22)$ \\
\hline $\mathrm{ADL}, \mathrm{n}=1241$ & $2.75[1.25-4.5]$ \\
\hline Loss of 1 or more ADL points in the last 3 months, $n=429$ & $86(20.05)$ \\
\hline Charlson comorbidity index, $\mathrm{n}=1086$ & $3[2-4]$ \\
\hline \multicolumn{2}{|l|}{ Ability to walk, $n=1202$} \\
\hline Yes, alone & $343(28.54)$ \\
\hline Yes, with human assistance & $162(13.48)$ \\
\hline Yes, with technical assistance & $315(26.21)$ \\
\hline No & $382(31.78)$ \\
\hline Fall during the month, $\mathrm{n}=1183$ & $228(19.27)$ \\
\hline Weight, $\mathrm{n}=1282$ & $61.87 \pm 14.41$ \\
\hline BMI, $n=1219$ & $24.18 \pm 5.16$ \\
\hline Albumin $(\mathrm{g} / \mathrm{l}), \mathrm{n}=1203$ & $34.02 \pm 4.66$ \\
\hline Malnutrition, $\mathrm{n}=1298$ & $821(63.25)$ \\
\hline \multicolumn{2}{|l|}{ Sensory deficit, $\mathrm{n}=933$} \\
\hline Yes, auditory & $183(19.61)$ \\
\hline Yes, visual & $183(19.61)$ \\
\hline Yes, auditory and visual & $150(16.08)$ \\
\hline No & $417(44.69)$ \\
\hline Number of drugs, $n=1295$ & $8.04 \pm 3.24$ \\
\hline \multicolumn{2}{|l|}{ At least one prescribing of : } \\
\hline - Neuroleptic, $n=1267$ & $291(22.95)$ \\
\hline - Benzodiazepine, $\mathrm{n}=1282$ & $745(57.07)$ \\
\hline - Antidepressant, $\mathrm{n}=1274$ & $665(52.12)$ \\
\hline MMSE score, $\mathrm{n}=640$ & 15 [9-19] \\
\hline BPSD, $n=1201$ & $513(42.71)$ \\
\hline NPI score, $\mathrm{n}=318$ & $34[20-51]$ \\
\hline Hospitalization within 3 months, $n=1169$ & $668(57.14)$ \\
\hline Scheduled hospitalization & $375(28.71)$ \\
\hline Hospitalization in emergency room & $336(25.72)$ \\
\hline Presence of bedsores, $n=1199$ & $211(17.60)$ \\
\hline Pain, $n=1217$ & $159(13.06)$ \\
\hline Short-GDS, $n=363$ & $0[0-2]$ \\
\hline Addressed directly by treating physician, $n=1221$ & $814(66.67)$ \\
\hline \multicolumn{2}{|c|}{$\begin{array}{l}\text { ADL, Activities of Daily Living }[0=\text { Low (patient very dependent), } 6=\text { High (patien } \\
\text { independent)]; BMI, Body Mass Index; GDS, Geriatric Depression Scale; g/l, gram pe } \\
\text { liter; MMSE, Mini Mental State; BPSD, Behavioural and Pyschological Symptoms o } \\
\text { Dementia; NPI, Neuropsychiatric Inventory; }\end{array}$} \\
\hline
\end{tabular}

\section{Discussion}

Reducing the inappropriate use of the emergency room for $\mathrm{NH}$ residents while meeting the expectations of caregivers faced with caring for residents is an important mission that hospital geriatric teams must assume. To our knowledge, our proposal of the responsive day hospital specifically dedicated to $\mathrm{NH}$ residents is a new innovative alternative that offers a practical response to the problem of inappropriate and potentially avoidable hospitalizations for $\mathrm{NH}$ residents.

Table 2

Reasons for hospitalization of residents hospitalized in the innovative day hospital dedicated to nursing home residents

\begin{tabular}{lcc}
\hline Reasons for hospitalization $(\mathbf{n = 1 9 1 8 )}$ & $\mathbf{n}$ & $\mathbf{\%}$ \\
\hline Cognitive disorders & 336 & 17.52 \\
Behavioral disorders & 297 & 15.48 \\
Bedsores and slow wound healing & 223 & 11.63 \\
Nutritional status & 197 & 10.27 \\
Neurology & 193 & 10.06 \\
Cardiology & 152 & 7.92 \\
Psychiatry & 71 & 3.70 \\
Assessment of fall & 65 & 3.39 \\
Internal medicine & 57 & 2.97 \\
Transfusion & 49 & 2.55 \\
Urology & 44 & 2.29 \\
Pain and palliative care & 33 & 1.72 \\
Botulinum toxin injection and monitoring & 25 & 1.30 \\
Hematology & 22 & 1.15 \\
Occupational therapy & 19 & 0.99 \\
Physical therapy & 15 & 0.78 \\
Pneumology & 14 & 0.73 \\
Reassessment after acute episode & 12 & 0.63 \\
Rheumatology & 12 & 0.63 \\
Dentist & 12 & 0.63 \\
Speech therapist & 10 & 0.52 \\
Endocrinology & 9 & 0.47 \\
Geriatric oncology & 7 & 0.36 \\
Gastroenterology & 7 & 0.36 \\
Other reason & 37 & 1.93 \\
\hline & & \\
\hline
\end{tabular}

Without demonstrating the efficiency of our system, our experimentation over a 2-year period suggests that, in many clinical situations, residents can be cared for without going to the emergency room and without being exposed to hospital iatrogenics (25). The occupancy rate of this new structure and the high number of NHs that regularly send their residents to it also testifies to its usefulness for $\mathrm{NH}$ care teams. Without such a system, NHs struggle to care for a variety of clinical situations which result in transfers to emergency rooms, in the absence of an alternative (11). Decisions to transfer $\mathrm{NH}$ residents to emergency rooms most often involve a conscious medical choice. Previous works showed us that the decisions to transfer decisions are usually made by a doctor $(72 \%$ of cases), and more often (65\%) during the day (26). Care with a medical and paramedical team specializing in geriatrics, short care times, easy access to specialist advice and a technical platform, without multiplying the movements of residents, 


\section{AN INNOVATIVE DAY HOSPITAL DEDICATED TO NURSING HOME RESIDENT}

appear in our action as a realistic alternative to meet the expectations of actors in the field. Characteristics of residents sent to the DH NH (Table 1) correspond to those of dependent, polypathological, elderly patients, regularly and recently hospitalized and thus at high risk of new transfers to emergency rooms. The reasons for sending patients are very often associated with dementia complications (particularly psychobehavioral disorders) and are problems primarily faced by NHs (2). The reasons correspond well to factors for inappropriately sending residents to emergency rooms suggesting that they have somatic decompensation falling within the emergency category and have been successfully transferred to emergency rooms. In other words, we believe that our strategy of sending residents directly to this $\mathrm{DH}$, which is the proper target of residents without causing missed opportunities. This is made possible by the organization of upstream care for emergency rooms, but also through a system allowing a response within short time periods.

The prevention of inappropriate hospitalizations for $\mathrm{NH}$ residents is a complex approach that can only be effective if it is based on multiple synchronized actions (25). The importance of training (27), motivation (28) of NH care teams, implementation of preventive measures, use of the assessment protocol before transferring to the emergency room (16), taking into account the opinion of the resident, drafting advance directives, and prior discussion with the families are all means which, in fine, help to curb the inappropriate use of emergency rooms. The INTERACT program developed in the United States focused on training the NH care team, but not involving all the levers of action, did not show its effectiveness by decreasing hospitalizations (16). Therefore, it seems important to associate these methods of training $\mathrm{NH}$ teams with an improvement of the healthcare sector for residents. A specificity of the DH NH is to provide care centered on the patient, adapted to the functional limitations of the resident, concerned about direct communication with the NH (all residents responded to the DH with a detailed letter by considering the quality of communication as an important factor in the flow of hospital-to-NH transitions) (29). This communication is also centered on preventing the most frequent causes of avoidable hospitalizations.

Thus, drug-induced iatrogenic disorder is a frequent cause of avoidable hospitalizations for NH residents (30). The study conducted by Lau et al. shows that residents who had at least one potentially inappropriate prescription had a significantly higher risk of hospitalization than residents who did not (OR, 1.27; p=0.002) (31). For all residents hospitalized in the $\mathrm{DH} \mathrm{NH}$, a hospital pharmacist made an analysis of their prescriptions. Proposals were given in the hospital discharge letter to improve the prescription in order to reduce druginduced iatrogenic disorder (32). We believe that this strategy has didactic virtues and therefore an impact that goes far beyond the single case of the assessed resident. The proposals aim to combat inappropriate prescriptions (overuse), allow for therapeutic adjustments (misuse), but are also the opportunity to give reminders of preventive measures (e.g. influenza and pneumococcal vaccine, vitamin D supplementation (33) (underuse). The relevance of this prescription revision is optimized by direct collaboration between the geriatrician and the pharmacist present within the DH.

The DH NH is also a real asset within a partnership with emergency room physicians, who can then respond to the problem of early readmissions to emergency rooms by directing $\mathrm{NH}$ residents to a DH in the days that follow, when traditional hospitalization after the transfer to an emergency room is not required.

We can also only exclude a proportion of residents passing through the DH NH inappropriately. However, no resident was in a situation falling under the category of a vital emergency. Furthermore, we believe that an improper transfer to the DH $\mathrm{NH}$ would not have unfavorable consequences like an improper transfer to an emergency room. The DH NH must not be substituted for any other types of care, such as telemedicine especially when moving the resident is not justified (Figure 1). It would therefore be interesting, in a later study, to verify whether the hospitalizations of residents in the $\mathrm{DH} \mathrm{NH}$ are appropriate.

\section{Figure 1}

Day hospital dedicated to nursing home residents within geriatric care network

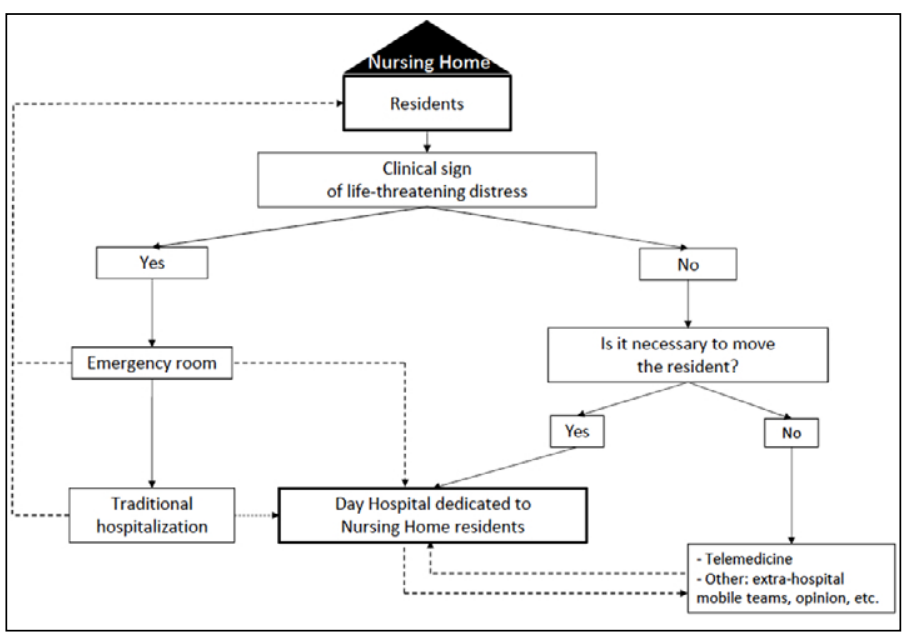

In conclusion, our experience over a 2-year period suggests that the innovative day hospital for $\mathrm{NH}$ residents could be a practical response to the problem of inappropriate and avoidable transfers of $\mathrm{NH}$ residents to emergency rooms. Care centered on the resident and adapted to his/her characteristics, minimizes the iatrogenic events linked to traditional hospitalization and allows the quick redress to advice from specialists and a technical platform in a short period of time while not multiplying the movements of the resident. We believe that this innovation could easily be utilized in other hospitals. 


\section{THE JOURNAL OF NUTRITION, HEALTH \& AGINGC}

Conflict of Interest: The authors have no conflict of interest to declare.

Ethical standards: The use of data concerning the patients was approved by the CNIL (Consultative Committee for Treatment of Research Information on Health) declaration of Toulouse University Hospital.

Open Access: This article is distributed under the terms of the Creative Commons Attribution 4.0 International License (http://creativecommons.org/licenses/by/4.0/), which permits use, duplication, adaptation, distribution and reproduction in any medium or format, as long as you give appropriate credit to the original author(s) and the source, provide a link to the Creative Commons license and indicate if changes were made.

\section{References}

1. Pearson KB, Coburn AF. Emergency Transfers of the Elderly from Nursing Facilities to Critical Access Hospitals: Opportunities for Improving Patient Safety and Quality. (Policy Brief \#32). Portland, ME: Flex Monitoring Team; January 2013

2. Rolland Y, Abellan van Kan G, Hermabessiere S, et al. Descriptive study of nursing home residents from the REHPA network. J Nutr Health Aging. 2009;13(8):679-683.

3. Burke RE, Rooks SP, Levy C, et al. Identifying Potentially Preventable Emergency Department Visits by Nursing Home Residents in the United States. J Am Med Dir Assoc. 2015;16(5):395-399. doi:10.1016/j.jamda.2015.01.076

4. Rothschild JM, Bates DW, Leape LL. Preventable Medical Injuries in Older Patients. Arch Intern Med. 2000;160(18):2717-2728. doi:10.1001/archinte.160.18.2717

5. Han JH., Morandi A, Ely EW, et al. Delirium in the Nursing Home Patients Seen in the Emergency Department. J Am Geriatr Soc. 2009;57(5):889-894. doi:10.1111/ j.1532-5415.2009.02219.x

6. Launay CP, Annweiler C, de Decker L, et al. Risk of in-hospital mortality following emergency department admission: results from the geriatric EDEN cohort study. J Nutr Health Aging. 2014;18(1):83-86. doi:10.1007/s12603-013-0038-3

7. Baum SA, Rubenstein LZ. Old people in the emergency room: age-related differences in emergency department use and care. J Am Geriatr Soc. 1987;35(5):398-404.

8. Casalino E, Wargon M, Peroziello A, et al. Predictive factors for longer length of stay in an emergency department: a prospective multicentre study evaluating the impact of age, patient's clinical acuity and complexity, and care pathways. Emerg Med J. 2014;31(5):361-368. doi:10.1136/emermed-2012-202155

9. Downing A, Wilson RC, Cooke MW. Which patients spend more than 4 hours in the Accident and Emergency department? J Public Health Oxf Engl. 2004;26(2):172-176. doi:10.1093/pubmed/fdh141

10. Kawano T, Nishiyama K, Anan H, et al. Direct relationship between aging an overcrowding in the $\mathrm{ED}$, and a calculation formula for demand projection: a crosssectional study. Emerg Med J. 2014;31(1):19-23. doi:10.1136/emermed-2012-202050

11. Ouslander JG, Lamb G, Perloe M, et al. Potentially avoidable hospitalizations of nursing home residents: frequency, causes, and costs: [see editorial comments by Drs. Jean F. Wyman and William R. Hazzard, pp 760-761]. J Am Geriatr Soc. 2010;58(4):627-635. doi:10.1111/j.1532-5415.2010.02768.x

12. Saliba D, Kington R, Buchanan J, et al. Appropriateness of the decision to transfer nursing facility residents to the hospital. J Am Geriatr Soc. 2000;48(2):154-163.

13. Walker JD, Teare GF, Hogan DB, et al. Identifying potentially avoidable hospita admissions from canadian long-term care facilities. Med Care. 2009;47(2):250-254 doi:10.1097/MLR.0b013e3181847588

14. Graverholt B, Forsetlund L, Jamtvedt G. Reducing hospital admissions from nursing homes: a systematic review. BMC Health Serv Res. 2014;14:36. doi:10.1186/14726963-14-36

15. Menand E, Lenain E, Lazarovici C, et al. French Multicenter Evaluation of the Appropriateness of Admission to the Emergency Department of the Over-80s. J Nutr Health Aging. 2015;19(6):681-687. doi:10.1007/s12603-015-0489-9
16. Kane RL, Huckfeldt P, Tappen R, et al. Effects of an Intervention to Reduce Hospitalizations From Nursing Homes: A Randomized Implementation Trial of the INTERACT Program. JAMA Intern Med. 2017;177(9):1257-1264. doi:10.1001/ jamainternmed 2017.2657

17. Trahan LM, Spiers JA, Cummings GG. Decisions to Transfer Nursing Home Residents to Emergency Departments: A Scoping Review of Contributing Factors and Staff Perspectives. J Am Med Dir Assoc. 2016;17(11):994-1005. doi:10.1016/j. jamda.2016.05.012

18. Rolland Y, Mathieu C, Piau C, et al. Improving the Quality of Care of LongStay Nursing Home Residents in France. J Am Geriatr Soc. 2016;64(1):193-199. doi:10.1111/jgs.13874

19. de Souto Barreto P, Lapeyre-Mestre M, Vellas B, et al. Multimorbidity type, hospitalizations and emergency department visits among nursing home residents: a preliminary study. J Nutr Health Aging. 2014;18(7):705-709. doi:10.1007/s12603014-0030-6

20. McGregor MJ, Abu-Laban RB, Ronald LA, et al. Nursing home characteristics associated with resident transfers to emergency departments. Can J Aging. 2014;33(1):38-48. doi:10.1017/S0714980813000615

21. Charlson ME, Pompei P, Ales KL, et al. A new method of classifying prognostic comorbidity in longitudinal studies: development and validation. J Chronic Dis 1987;40(5):373-383.

22. Katz S, Ford AB, Moskowitz RW, et al. Studies of illness in the aged. The index of ADL : a standardized measure of biological and psychosocial function. JAMA 1963;185:914-919.

23. Haute Autorité de Santé. Stratégie de prise en charge en cas de dénutrition protéinoénergétique chez la personne âgée. Recommandations professionnelles. 2007. https://www.has-sante.fr/portail/upload/docs/application/pdf/denutrition_personne_ agee_2007_-_recommandations.pdf. Accessed 26 June 2018.

24. Cummings JL, Mega M, Gray K, et al. The Neuropsychiatric Inventory: comprehensive assessment of psychopathology in dementia. Neurology 1994;44(12):2308-2314

25. Ouslander JG, Berenson RA. Reducing unnecessary hospitalizations of nursing home residents. N Engl J Med. 2011;365(13):1165-1167. doi:10.1056/NEJMp1105449

26. Rolland Y, Andrieu S, Crochard A, et al. Psychotropic Drug Consumption at Admission and Discharge of Nursing Home Residents. J Am Med Dir Assoc. 2012;13(4):407.e7-407.e12. doi:10.1016/j.jamda.2011.12.056

27. Kirsebom M, Hedström M, Pöder U, et al. Transfer of nursing home residents to emergency departments: organizational differences between nursing homes with high vs. low transfer rates. Nurs Open. 2017;4(1):41-48. doi:10.1002/nop2.68

28. Cohen AB, Knobf MT, Fried TR. Avoiding Hospitalizations From Nursing Home for Potentially Burdensome Care: Results of a Qualitative Study. JAMA Intern Med 2017;177(1):137-139. doi:10.1001/jamainternmed.2016.7128

29. King BJ, Gilmore-Bykovskyi AL, Roiland RA, et al. The Consequences of Poor Communication during Hospital to Skilled Nursing Facility Transitions: A Qualitative Study. J Am Geriatr Soc. 2013;61(7). doi:10.1111/jgs.12328

30. Narbey D, Jolly D, Mahmoudi R, et al. Relationship between anticholinergic drug use and one-year outcome among elderly people hospitalised in medical wards via emergency department: the SAFES cohort study. J Nutr Health Aging. 2013;17(9):766-771. doi:10.1007/s12603-013-0349-4

31. Lau DT, Kasper JD, Potter DEB, et al. Hospitalization and death associated with potentially inappropriate medication prescriptions among elderly nursing home residents. Arch Intern Med. 2005;165(1):68-74. doi:10.1001/archinte.165.1.68

32. Rouch L, Farbos F, Cool C, et al. Hospitalization Drug Regimen Changes in Geriatric Patients and Adherence to Modifications by General Practitioners in Primary Care. J Nutr Health Aging. 2018;22(3):328-334. doi: 10.1007/s12603-017-0940-1

33. Beauchet $\mathrm{O}$, Launay $\mathrm{C}$, de Decker L. et al. Who is at risk of long hospital stay among patients admitted to geriatric acute care unit? Results from a prospective cohort study. J Nutr Health Aging. 2013;17(8):695-9. doi: 10.1007/s12603-013-0333-z 